\title{
NEW COLOR FILTER ARRAYS OF HIGH LIGHT SENSITIVITY AND HIGH DEMOSAICKING PERFORMANCE
}

\author{
Jue Wang ${ }^{1}$, Chao Zhang ${ }^{1 *}$, Pengwei Hao ${ }^{1,2 \dagger}$ \\ ${ }^{1}$ Key Laboratory of Machine Perception, Peking University, Beijing 100871, China \\ ${ }^{2}$ School of Electronic Engineering and Computer Science, Queen Mary, \\ University of London, London E1 4NS, U.K. \\ ${ }^{*}$ chzhang@cis.pku.edu.cn, †phao@eecs.qmul.ac.uk
}

\begin{abstract}
For high light sensitivity, new CFA designs use panchromatic pixels, aka white pixels, that no visible spectrum energy is filtered. Kodak's CFA2.0 has 50\% white pixels, but the demosaicking performance is not good. We present in this work a set of new color filter arrays (CFA) of high light sensitivity and high demosaicking performance which were obtained by using a CFA design methodology in the frequency domain. The new patterns are of size $5 \times 5$ and come from the same frequency structure, which has one luma in the base band at $(0,0)$ and four chromas (two conjugate pairs) placed at $(4 \pi / 5,2 \pi / 5),(-4 \pi / 5,-2 \pi / 5),(2 \pi / 5,-4 \pi / 5)$ and $(-2 \pi / 5,4 \pi / 5)$, respectively. The new patterns are optimized to have only white (panchromatic) and three primary color pixels and the pixels are found to be $40 \%$ white, $20 \%$ red, $20 \%$ green and $20 \%$ blue by pixel color constrained optimization. Our demosaicking experiments show that our new CFA patterns outperform Kodak CFA2.0 in both objective and subjective quality.
\end{abstract}

Index Terms - Color Filter Array (CFA), Light Sensitivity, Discrete Fourier Transform (DFT), Multiplexing, Demosaicking

\section{INTRODUCTION}

Nowadays, almost every modern camera, every video camera, every camera phone, every machine vision system and every scanner has a color filter array (CFA) fabricated on top of the light sensors for creation of the color image. The CFA-filtered images acquire energy of only one color light at each pixel and a technique known as "demosaicking" $[1,2,3]$ follows to reconstruct the images with all the three primary colours, red, green and blue, at each pixel. Except some random CFAs [4, 5], a two-dimensional CFA design generally comprises a plurality of minimal repeating patterns tiled to cover the entire array of image sensors. Different minimal repeating CFA patterns characterise different CFAs. Since Bayer of Kodak invented the first color filter array in 1976 [6] by using 1/2 green, 1/4 red and $1 / 4$ blue pixels, not many CFA patterns have been proposed until recent years. Most patterns [7, 8, 9] were proposed for better demosaicking performance. Recently, high light sensitivity cameras are highly expected, which can be used in low-light environment and for high-speed imaging. In 2007, Kodak published its new generation of CFA [10], called CFA2.0. Kodak's CFA2.0 (Kodak CFA) uses $1 / 4$ green, $1 / 8$ red and $1 / 8$ blue pixels and $1 / 2$ white pixels (also known as panchromatic pixels, wide-band pixels, luminance pixels)

This work is supported by research funds of NBRPC No.2011CB302400 and NSFC No.61071156. which allow no visible spectrum energy being filtered and have the highest light sensitivity. However, there is a big problem with the new high light sensitivity CFA, Kodak CFA: The demosaicking performance as published so far is much worse than Bayer CFA and than we expected.

The goal of this work is to find some new color filter arrays of high light sensitivity and higher demosaicking performance than Kodak CFA. Based on the CFA design methodology [11, 12, 13], we employ a new frequency structure corresponding to 5x5 CFA patterns to find a set of new CFA patterns with only white (W), red $(\mathrm{R})$, green $(\mathrm{G})$ and blue (B) pixels. The benchmark demosaicking methods we used are a frequency domain filtering method evolved from Dubois's method [14] and a universal demosaicking method, known as a generic variational approach [15], which provides a robust demosaicking framework for solving the optimization problem as a large sparse system of linear equations.

The remainder of this paper is organized as follows. Section 2 provides a brief review of the CFA representation. Section 3 gives the details of our new CFA design and optimization, including the frequency structure, the pixel color constraints for CFAs with only white and primary color pixels, and our new 5x5 CFA patterns. In Section 4, we present the associated demosaicking method for our new CFAs. In Section 5, the experimental results are presented to show the comparison between the Kodak CFA and our newly proposed CFA patterns. In section 5, we draw the concluding remarks.

\section{CFA REPRESENTATION}

In this section, we briefly show two representations of a CFA pattern in the spatial and the frequency domain: the CFA pattern and the frequency structure [13]. Actually, they constitute a symbolic Discrete Fourier Transform (DFT) pair.

\subsection{CFA Pattern}

A periodic CFA is a periodic mosaic of spectrally selective filters, and the minimal repeating CFA pattern is known as a CFA pattern. Every rectangular CFA pattern can be represented by a matrix that consists of the R, G and B primary colors or combination of them. For example, the Bayer pattern can be represented by a matrix as in $\mathrm{Eq}(1)$ :

$$
h_{p, \text { Bayer }}=\left[\begin{array}{cc}
G & R \\
B & G
\end{array}\right]
$$

Similarly, the CFA pattern of Kodak CFA [10] is Eq(2), also 
shown in Fig. 1(a) with padding the primary colors and white pixels:

$$
h_{p, \text { Kodak }}=\left[\begin{array}{cccc}
W & B & W & G \\
B & W & G & W \\
W & G & W & R \\
G & W & R & W
\end{array}\right]
$$

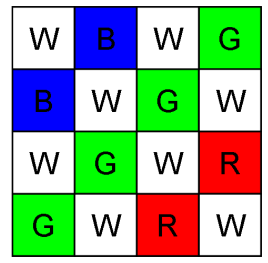

(a) Kodak CFA

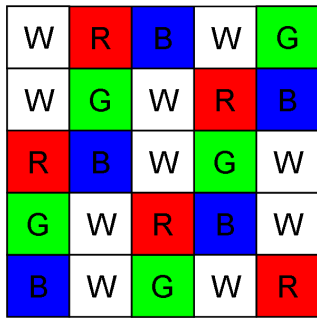

(c) Proposed CFA

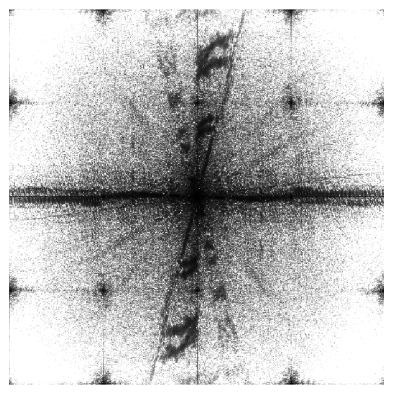

(b) Spectrum with (a)

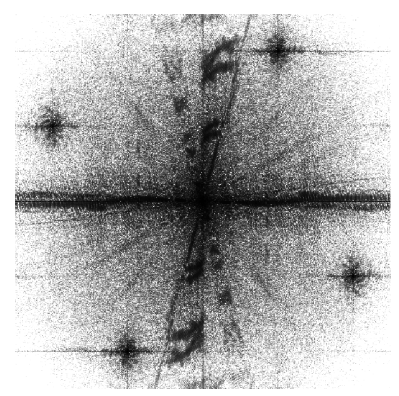

(d) Spectrum with (c)
Fig. 1. CFA patterns and the log-magnitude spectra of the CFAfiltered images for lighthouse.

\subsection{Frequency Structure}

As we know, the frequency spectrum of a CFA-filtered image is made up of a luminance component (luma) at base frequency band, and some chrominance components (chromas) at high frequency bands $[2,14]$. The frequency structure is a matrix to locate all those components and to give the composition of each component, so the frequency structure of a CFA is intuitive and visual for CFA analysis. The frequency structure of a CFA can be obtained by computing the symbolic DFT of the CFA pattern. For example, the frequency structure of the Bayer CFA is as in $\mathrm{Eq}(3)$ :

$$
\begin{aligned}
S_{\text {Bayer }} & =D F T\left[h_{p, \text { Bayer }}\right]=D F T\left[\begin{array}{ll}
G & R \\
B & G
\end{array}\right] \\
& =\frac{1}{4}\left[\begin{array}{cc}
R+2 G+B & -R+B \\
R-B & -R+2 G-B
\end{array}\right]
\end{aligned}
$$

There are one luma $(R+2 G+B) / 4$ at baseband, and three chromas, $(-R+2 G-B) / 4,(R-B) / 4$ and $(-R+B) / 4$ (denoted by $F_{L}$, $F_{C 1}, F_{C 2}$ and $-F_{C 2}$, respectively) in $S_{\text {Bayer }}$.

Likewise, applying the symbolic DFT to the Kodak CFA pattern (2), we can find the frequency structure of Kodak CFA as in Eq(4):

$$
S_{\text {Kodak }}=\left[\begin{array}{cccc}
F_{L} & F_{C 3}^{*} & 0 & F_{C 3} \\
F_{C 3}^{*} & F_{C 2}^{*} & -F_{C 3} & 0 \\
0 & -F_{C 3} & F_{C 1} & -F_{C 3}^{*} \\
F_{C 3} & 0 & -F_{C 3}^{*} & F_{C 2}
\end{array}\right]
$$

where * denotes the complex conjugate. There are totally twelve components in the frequency structure $\operatorname{Eq}(4)$, one luma in the baseband at $(0,0)$, one corner chroma in the highest frequency band at $(\pi, \pi)$ and five conjugate pairs of chromas are distributed in other bands in the frequency spectrum plane. Obviously, although it has $50 \%$ white pixels which provides the higher light sensitivity than Bayer CFA and other CFAs with no or less white pixels, the aliasing problem is quite serious for Kodak CFA-filtered images. This also explains why the demosaicking performance with Kodak CFA cannot be so favorable.

With a frequency structure, we can easily find a linear relationship between the luminance and chrominance components and the images of the primary colors, as in $\mathrm{Eq}(5)$ :

$$
\left[\begin{array}{c}
F_{L} \\
F_{C 1} \\
F_{C 2} \\
\vdots
\end{array}\right]=T \cdot\left[\begin{array}{c}
R \\
G \\
B
\end{array}\right]
$$

where $T$ is called the multiplexing matrix, which gives the specific composition of each component multiplexed from the image spectra of the primary colors, $\mathrm{R}, \mathrm{G}$ and $\mathrm{B}$.

Reversely, we can find the demosaicking matrix by the inverse or pseudo-inverse of the multiplexing matrix: $D=T^{-1}$ if $T$ is invertible, or $D=T^{+}$if $T$ is not square. Then the RGB images can be obtained from the multiplexed components for demosaicking in $\mathrm{Eq}(6)$ :

$$
\left[\begin{array}{c}
R \\
G \\
B
\end{array}\right]=D \cdot\left[\begin{array}{c}
F_{L} \\
F_{C 1} \\
F_{C 2} \\
\vdots
\end{array}\right]
$$

\section{CFA DESIGN}

Motivated by the high light sensitivity of Kodak CFA resulting from all-pass white pixels, we add a number of white pixels as a constraint in our CFA design framework to find new CFAs of both high light sensitivity and high demosaicking performance.

\subsection{Specification of New Frequency Structure}

Observation of the frequency structures and general image spectra tells that: 1) the fewer chromas, the less frequency aliasing; 2) the farther the distances between luma and chromas and between chromas, the less frequency aliasing; 3) another factor that influences the accuracy of demosaicked images of the primary colors is from the inverse transform from multiplexed components to the primary colors: the less the norm of demosaicking matrix $D$, the better the demosaicking performance.

First, We choose a good frequency structure for less aliasing. Especially we determine the locations of all the chromas for less aliasing among luma and chromas and specify some relations between the chromas, some of which may be conjugate to each other. Balancing the design expectations, we choose a new $5 \times 5$ frequency structure with two conjugate pairs of chromas for our CFA design, as shown in $\operatorname{Eq}(7)$ :

$$
S_{\text {new } C F A s}=\left[\begin{array}{ccccc}
F_{L} & 0 & 0 & 0 & 0 \\
0 & 0 & F_{C 2} & 0 & 0 \\
0 & 0 & 0 & 0 & F_{C 1}^{*} \\
0 & F_{C 1} & 0 & 0 & 0 \\
0 & 0 & 0 & F_{C 2}^{*} & 0
\end{array}\right]
$$


It has one luma in the base band at $(0,0)$ and four chromas (two conjugate pairs) placed at $(4 \pi / 5,2 \pi / 5),(-4 \pi / 5,-2 \pi / 5)$, $(2 \pi / 5,-4 \pi / 5)$ and $(-2 \pi / 5,4 \pi / 5)$, respectively. The two conjugate pairs of chromas $\left(F_{C 1}, F_{C 1}^{*}\right.$ and $\left.F_{C 2}, F_{C 2}^{*}\right)$ in the newly designed frequency structure are centrally symmetric to each other in the frequency plane, which corresponds to the central symmetry property of discrete Fourier transform.

With the frequency structures $\mathrm{Eq}(7)$ and $\mathrm{Eq}(3)$, we can easily find that the minimum distance between luma and chromas of our CFA $\left(2 \pi \cdot \sqrt{(1 / 5)^{2}+(2 / 5)^{2}} \approx 0.894 \pi\right)$ is much larger than that of Kodak CFA $(2 \pi / 4=0.5 \pi)$, so there must be less aliasing for our CFA both in theory and in practice and the demosaicking error can be less consequently.

\subsection{Optimization}

Based on the above frequency structure, $\mathrm{Eq}(7)$, the CFA design involves the solution of a constrained optimization problem that aims at minimizing the demosaicking error. In order to find CFAs with a number of white pixels and some pixels of only primary colors, we use the geometric design method [13] with an additional constraint for pixel colors in the spatial domain for optimization:

$$
\forall(i, j), h_{p, \text { new } C F A s}(i, j) \in\{W, R, G, B\}
$$

Then we find the multiplexing coefficients of our new $5 \times 5$ frequency structure:

$$
\left\{\begin{aligned}
F_{L}= & (R+G+B) / 3 \\
F_{C 1}= & ((\sqrt{5}-i \sqrt{25-2 \sqrt{5}}) R+\sqrt{5}(1+i \sqrt{5+2 \sqrt{5}}) G \\
& -(2 \sqrt{5}+i \sqrt{10-2 \sqrt{5}}) B) / 30, \\
F_{C 2}= & (-(\sqrt{5}+i \sqrt{25+2 \sqrt{5}}) R+i \sqrt{5}(i+\sqrt{5-2 \sqrt{5}}) G \\
& +(2 \sqrt{5}+i \sqrt{2(5+\sqrt{5})}) B) / 30 .
\end{aligned}\right.
$$

With such coefficients, we can find the multiplexing and the demosaicking matrices as in $\operatorname{Eq}(11)$ and $D=T^{-1}$.

Applying the symbolic inverse DFT to the new frequency structure, we obtain a new 5x5 CFA pattern, as shown in Eq(10) and Fig. $1(\mathrm{c})$.

$$
h_{p, \text { new CFA }}=\left[\begin{array}{ccccc}
W & R & B & W & G \\
W & G & W & R & B \\
R & B & W & G & W \\
G & W & R & B & W \\
B & W & G & W & R
\end{array}\right]
$$

The new pattern has $40 \%$ white, $20 \%$ red, $20 \%$ green and $20 \%$ blue pixels. If we use all the 6 permutations of RGB to replace the RGB colors in the above new pattern and transpose the patterns, we can have 11 more patterns. Thus, we can find a set of 12 new CFA patterns with the same optimal frequency structure.

\section{DEMOSAICKING}

Given the frequency structure of a CFA pattern, we can design some filters to separately obtain the luma and all the chromas in a CFAfiltered image in frequency domain by convolution with the filters at the corresponding frequency positions. With the multiplexing coefficients for each component found by optimization, just like Dubois demosaicked Bayer CFA sampled images [14], we can then use the linear transform $\mathrm{Eq}(6)$ to find $\mathrm{R}, \mathrm{G}, \mathrm{B}$ values from the luma and chromas.
Table 1. Performance comparison with two demosaicking algorithms. The average CPSNR is calculated with $10 \log$ (average MSE).

\begin{tabular}{|c|c|c|c|c|}
\hline & \multicolumn{2}{|c|}{ Dubois's method [14] } & \multicolumn{2}{c|}{ Condat's method [15] } \\
\hline Image & Kodak & Proposed & Kodak & Proposed \\
\hline 1 & 23.18 & $\mathbf{3 5 . 2 3}$ & 34.21 & $\mathbf{3 5 . 3 5}$ \\
2 & 30.94 & $\mathbf{3 7 . 4 9}$ & 36.80 & $\mathbf{3 8 . 1 1}$ \\
3 & 32.68 & $\mathbf{3 7 . 4 6}$ & 38.23 & $\mathbf{3 8 . 4 0}$ \\
4 & 32.75 & $\mathbf{3 7 . 3 2}$ & 36.95 & $\mathbf{3 8 . 3 2}$ \\
5 & 25.89 & $\mathbf{3 2 . 9 6}$ & 32.41 & $\mathbf{3 3 . 6 2}$ \\
6 & 25.34 & $\mathbf{3 6 . 5 6}$ & 35.52 & $\mathbf{3 6 . 7 4}$ \\
7 & 31.71 & $\mathbf{3 8 . 2 2}$ & 37.47 & $\mathbf{3 8 . 8 3}$ \\
8 & 22.21 & $\mathbf{3 3 . 2 8}$ & 31.69 & $\mathbf{3 3 . 3 7}$ \\
9 & 29.50 & $\mathbf{3 8 . 7 8}$ & 38.13 & $\mathbf{3 9 . 3 3}$ \\
10 & 32.00 & $\mathbf{3 6 . 6 3}$ & $\mathbf{3 8 . 8 7}$ & 38.12 \\
11 & 27.56 & $\mathbf{3 6 . 1 3}$ & 35.62 & $\mathbf{3 6 . 7 7}$ \\
12 & 31.62 & $\mathbf{4 0 . 1 8}$ & 39.68 & $\mathbf{4 0 . 6 1}$ \\
13 & 23.52 & $\mathbf{3 0 . 9 1}$ & 31.37 & $\mathbf{3 1 . 7 5}$ \\
14 & 27.21 & $\mathbf{3 2 . 2 4}$ & 32.01 & $\mathbf{3 3 . 3 0}$ \\
15 & 32.86 & $\mathbf{3 6 . 2 9}$ & 36.83 & $\mathbf{3 7 . 6 0}$ \\
16 & 28.81 & $\mathbf{3 9 . 5 8}$ & 39.20 & $\mathbf{3 9 . 8 9}$ \\
17 & 32.25 & $\mathbf{3 7 . 9 0}$ & 38.09 & $\mathbf{3 9 . 1 1}$ \\
18 & 27.17 & $\mathbf{3 3 . 8 7}$ & 33.91 & $\mathbf{3 4 . 8 3}$ \\
19 & 24.37 & $\mathbf{3 7 . 5 3}$ & 35.72 & $\mathbf{3 7 . 6 3}$ \\
20 & 30.57 & $\mathbf{3 6 . 7 1}$ & 37.33 & $\mathbf{3 8 . 0 2}$ \\
21 & 26.13 & $\mathbf{3 6 . 1 4}$ & 35.14 & $\mathbf{3 6 . 6 9}$ \\
22 & 29.25 & $\mathbf{3 5 . 5 9}$ & 35.33 & $\mathbf{3 6 . 5 5}$ \\
23 & 32.59 & $\mathbf{3 8 . 8 6}$ & 37.29 & $\mathbf{3 9 . 2 0}$ \\
24 & 26.60 & $\mathbf{3 1 . 9 0}$ & 33.01 & $\mathbf{3 3 . 2 3}$ \\
\hline Avg. & 27.30 & $\mathbf{3 5 . 4 2}$ & 35.17 & $\mathbf{3 6 . 1 9}$ \\
\hline & & & & \\
\hline
\end{tabular}

For more than two linear independent chromas, we can find two independent chromas for demosaicking such that the norm of the demosaicking matrix is the smallest, which makes demosaicking more stable and more accurate. Finally, all the R, G and B pixel values can be found with the demosaicking matrix and the corresponding components.

Another demosaicking method we use to assess our new CFAs is a generic variational approach [15], which is universal for arbitrary CFA patterns.

\section{EXPERIMENTS AND PERFORMANCE ANALYSIS}

As we expect for optimal CFA design, images sampled with our new CFAs show much less aliasing than with Kodak CFA in the frequency domain as shown in Fig. 1(b) and (d). We apply Dubois's frequency-domain demosaicking method [14] and Condat's generic variational demosaicking method [15] to both Kodak CFA and our new CFA for the demosaicking performance comparison. The experimental results with 24 Kodak color test images are listed in Table 1.

Table 1 shows that with Duboiss demosaicking method the proposed CFA performs much better (over 8dB higher) than Kodak CFA in average, and with Condats demosaicking method it can give even better performance and also $1 \mathrm{~dB}$ better than Kodak CFA.

The demosaicked images for test image lighthouse are partially displayed in Fig. 2. The images also tell that our 5x5 CFA is much better than Kodak CFA although the light sensitivity with $40 \%$ white pixels is a bit lower than Kodak CFA with $50 \%$ white pixels. 


$$
T=\left[\begin{array}{ccc}
1 / 3 & 1 / 3 & 1 / 3 \\
(\sqrt{5}-i \sqrt{25-2 \sqrt{5}}) / 30 & \sqrt{5}(1+i \sqrt{5+2 \sqrt{5}}) / 30 & -(2 \sqrt{5}+i \sqrt{10-2 \sqrt{5}}) / 30 \\
-(\sqrt{5}+i \sqrt{25+2 \sqrt{5}}) / 30 & i \sqrt{5}(i+\sqrt{5-2 \sqrt{5}}) / 30 & (2 \sqrt{5}+i \sqrt{2(5+\sqrt{5})}) / 30
\end{array}\right]
$$

\section{CONCLUSION}

Specifying a less aliasing frequency structure, using an additional pixel color constraint, and applying the optimal CFA design methodology, we have obtained a set of new CFAs of high light sensitivity, whose minimal repeating patterns have a size of $5 \times 5$ pixels, including $40 \%$ white, $20 \%$ red, $20 \%$ green and $20 \%$ blue pixels. Our demosaicking experiments with two methods demonstrate that our new CFA outperforms Kodak's CFA2.0. Thus, the new CFA patterns have both high light sensitivity and high demosaicking performance. Anyway, we do not know yet whether there are CFAs that have both higher demosaicking performance and higher light sensitivity with more than $50 \%$ white pixels, which merits further investigation.

\section{ACKNOWLEDGEMENT}

The authors give thanks to E. Dubois of University of Ottawa and L. Condat of GREYC Lab for providing the source codes for demosaicking.

\section{REFERENCES}

[1] B. K. Gunturk, J. Glotzbach, Y. Altunbasak, R. W. Schaffer, and R. M. Mersereau, "Demosaicking: Color filter array interpolation," IEEE Signal Processing Mag., vol. 22, pp. 44-54, Jan. 2005.

[2] D. Alleyson, S. Susstrunk, and J. Herault, "Linear demosaicing inspired by the human visual system," IEEE Trans. Image Processing, vol. 14, pp. 439-449, Apr. 2005.

[3] O. Losson, L. Macaire, and Y. Yang, Comparison of Color Demosaicing Methods, vol. 162, chapter 5 in Advances in Imaging and Electron Physics, pp. 173-265, 2010.

[4] W. Zhu, K. Parker, and M.A. Kriss, "Color filter arrays based on mutually exclusive blue noise patterns," J. Vis. Commun. Image Represent., vol. 10, no. 3, pp. 245-267, Sept. 1999.

[5] L. Condat, "A new random color filter array with good spectral properties," in Proc. IEEE Internat. Conf. Image Process., Nov. 2009.

[6] B.E. Bayer, “Color imaging array," US Patent 3971 065, 1976.

[7] R. Lukac and K.N. Plataniotis, "Color filter arrays: design and performance analysis," IEEE Trans. Consumer Electron., vol. 51, no. 4, pp. 1260-1267, Nov. 2005.

[8] K. Hirakawa and P.J. Wolfe, "Spatio-spectral color filter array design for optimal image recovery," IEEE Trans. Image Processing, vol. 17, pp. 1876-1890, Oct. 2008.

[9] L. Condat, "A new color filter array with optimal sensing properties," in Proc. IEEE Internat. Conf. Image Process., Nov. 2009.

[10] J. Compton and J. Hamilton, "Image sensor with improved light sensitivity," US Patent 20070024 031A1, 2005.

[11] Y. Li, P. Hao, and Z. Lin, "Color filter arrays: representation and analysis," Tech. Rep. RR-08-04, Dept. of Computer Science, Queen Mary, Univ. of London (QMUL), 2008.
[12] Y. Li, P. Hao, and Z. Lin, "Color filter arrays: a design methodology," Tech. Rep. RR-08-03, Dept. of Computer Science, Queen Mary, Univ. of London (QMUL), 2008.

[13] P. Hao, Y. Li, Z. Lin, and E. Dubois, "A geometric method for optimal design of color filter arrays," IEEE Trans. Image Processing, in press, 2011.

[14] E. Dubois, "Frequency-domain methods for demosaicking of Bayer-sampled color images," IEEE Signal Processing Lett., vol. 12, pp. 847-850, Dec. 2005.

[15] L. Condat, "A generic variational approach for demosaicking from an arbitrary color filter array," in Proc. IEEE Internat. Conf. Image Process., Nov. 2009.

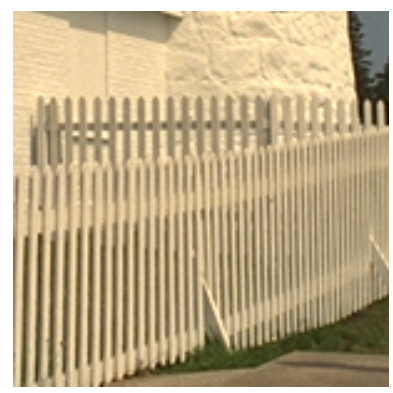

Original image

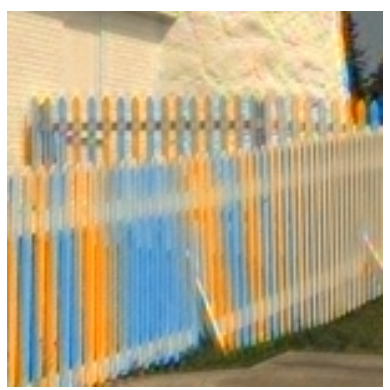

(a) Kodak CFA with [14]

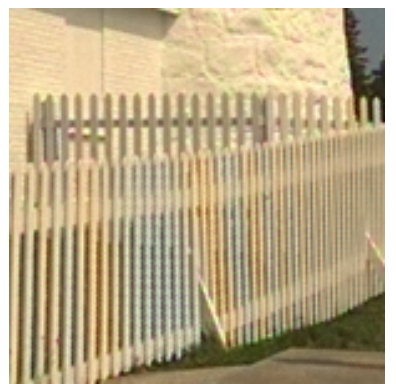

(c) Kodak CFA with [15]

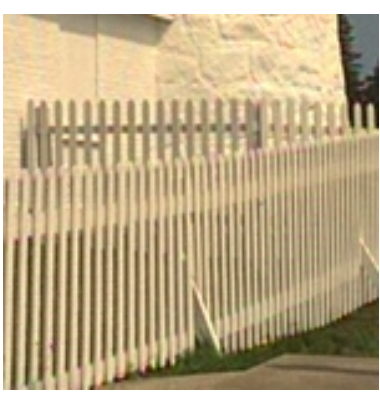

(b) Proposed CFA with [14]

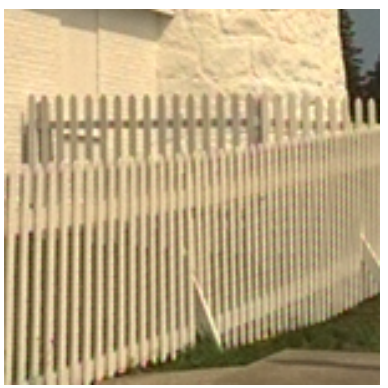

(d) Proposed CFA with [15]
Fig. 2. Part of the demosaicked image lighthouse. The demosaicking methods used: (a,b) Dubois's [14], (c,d) Condat's [15]. 